\title{
Potere e Politica Nell'esperienza Costituzionale del Brasile Postcoloniale. UnA RIFLESSIONE A PARTIRE DA UN'OPERA DI MACHADO DE ASSIS
}

Jacopo Paffarini*

1 L'Europa, le ex-colonie e la globalizzazione. 2 Machado de Assis e la società brasiliana tra impero e repubblica. 3 Un romanzo "di rottura." 4 La piaga dello schiavismo. 5 Una «modernizzazione conservatrice». Riferimenti.

\section{RIASSUNTO}

La complessità delle relazioni di potere è ascrivibile a molte opere di Machado de Assis, attraverso i suoi scritti si accede ad un ritratto della società carioca in un'epoca in cui lo Stato brasiliano era ancora alla ricerca di un definitivo assetto istituzionale. La posizione di Machado de Assis acquista in questo senso un duplice rilievo per lo studioso europeo: da un lato, costituisce una testimonianza delle tensioni sviluppatesi intorno ai processi di diffusione oltreoceano del pensiero politico e filosofico del Vecchio Continente; dall'altro, identifica la realtà brasiliana come il prodotto di questa contraddizione tra l'arcaico e il moderno, tra i canoni antichi e il progresso guidato dell'ambizione individuale.

Parole-chiave: Diritto e letteratura. Studi Post-coloniali. Dialogo accademico Brasile-Italia. Circolazione e recezione modelli giuridici occidentali.

\section{L'EUROPA, LE EX-COLONIE E LA GLOBALIZZAZIONE}

Nel corso degli ultimi decenni le esperienze di collaborazione accademica tra Italia e Brasile hanno promosso un avvicinamento tra due paesi comunemente ritenuti lontani, evidenziando una serie di legami culturali, storici ed economici a cui non sempre era stata concessa un'opportuna considerazione. Con la moltiplicazione dei luoghi e dei momenti di dialogo, inoltre, sono emersi nuovi punti di vista attraverso i quali è stato possibile

* Pós-Doutor em Direito Público (Faculdade Meridional IMED). Doutor em Direito Público (Università degli Studi di Perugia/Itália). Professor Permanente no Programa de Pós-Graduação Stricto Sensu em Direito, Faculdade Meridional - IMED. Bacharel em Direito pela Università degli Studi di Perugia. Pesquisador Visitante do Instituto Max-Planck de Direito Público Comparado - Heidelberg (Alemanha). E-mail: <jacopo.paffarini@imed.edu.br>. 
riorganizzare un bilancio delle esperienze e delle narrazioni diffuse relative al processo di civilizzazione sudamericano.

Nel presente Convegno Italo-Brasiliano è stato giustamente scelto un percorso ampio ed interdisciplinare, nella consapevolezza che i "punti di contatto" possono affermarsi anche in opposizione alle conoscenze maturate fino ad oggi nei vari settori accademici. D'altronde si tratta di una sfida non più rimandabile, tenuto conto che in ogni area delle scienze sociali è finalmente emerso il bisogno di "superare i confini" della tradizione scientifica del mondo occidentale.

Gli effetti disgreganti attribuiti all'intensificarsi dei flussi di circolazione di persone, idee, commodities e denaro esprimono infatti una crisi che colpisce, oltre alla "sovranità nazionale", gli stessi modelli istituzionali prodotti dal pensiero occidentale. Il ritorno dei particolarismi sociali, delle istanze localistiche, la sfiducia verso la democrazia rappresentativa identificata con il sistema dei partiti e, non ultimo, il prepotente ritorno dell'intolleranza razziale sono generalmente (e sbrigativamente) descritti come le conseguenze di un processo di globalizzazione che ha dato attenzione solamente ai mercati. ${ }^{1}$ Ancor prima del recente consolidamento di una dimensione transnazionale dei rapporti economici e comunicativi, tuttavia, gran parte di queste problematiche erano già emerse, seppur separatamente, in diverse aree del pianeta. Si dovrebbe perciò sostenere che la globalizzazione ha promosso, in alcuni casi, la loro "emigrazione", in altri, soltanto la generalizzazione del disagio ad esse legato.

A partire da queste considerazioni la continuazione di un dialogo accademico può servire, se non a tracciare delle soluzioni comuni, perlomeno a sollevare le questioni in un'ottica non più limitata da un solo sistema di pensiero, ma plurale e partecipata. Non si vuole ritornare sulle accuse di "eurocentrismo" avanzate in più occasioni nei confronti degli studi europei, soprattutto nel settore delle scienze sociali. ${ }^{2}$ Piuttosto si vuole richiamare l'attenzione sulla circolazione di idee che ha sempre unito il "centro" alla "periferia" del sistema produttivo capitalista e sull'inevitabile "manipolazione" a cui le stesse vengono sottoposte nel processo di ricezione e di adattamento alla nuova realtà ricevente. ${ }^{3}$

La civilizzazione del Brasile assume una rilevanza particolare in questo discorso per una serie di peculiarità del suo percorso storico. In primo luogo, l'insediamento della Corona Portoghese a Rio de Janeiro, durante il periodo coloniale, contribuì ad intensificare l'influenza europea nel paese latinoamericano, che diventò un punto di passaggio per molti artisti e scrittori del Vecchio Continente. In secondo luogo, il Brasile è una nazione che non ha mai smesso di ricevere "stranieri" (siano essi conquistatori, pionieri, schiavi, migranti in fuga dalla carestia e dalle guerre europee o, infine, investitori). Questo contribuisce alla sua complessità, alla diversificazione estrema delle condizioni di vita, alla conflittualità esistente sulla proprietà delle terre tra autoctoni (indigeni) e colonizzatori delle diverse generazioni, a concentrare le rivendicazioni in senso geografico (Nordest contro Sudest; élite agrarie dell'entroterra di discendenza italiana e tedesca contro le élite della costa atlantica di discendenza portoghese; 
indigeni dell'Amazzonia di oggi contro l'avanzamento delle città industriali e delle metropoli).

Le asimmetrie del processo di modernizzazione del Brasile sono ovviamente riconducibili alla varietà del suo tessuto sociale, dove i modelli della cultura occidentale si sono insediati passando attraverso importanti fasi di adattamento, che ne hanno determinato, per così dire, una "brasilianizzazione". È nell'ambito di quest'ultimo passaggio che i processi di trapianto degli istituti occidentali hanno manifestato le maggiori difficoltà, spesso deludendo le aspettative. ${ }^{4} \mathrm{Si}$ considerino, a titolo di esempio, le specificità del presidenzialismo brasiliano: la tipica concertazione delle funzioni di indirizzo politico presso il Capo dello Stato è "corretta" dalla frammentazione prodotta dalla scelta di un sistema proporzionale puro per l'elezione dei membri del Congresso. L'effetto rappresentativo è dunque compreso tra due tendenze asimmetriche, con l'instaurazione di equilibri notevolmente complessi dentro le alleanze di governo ed il rischio immanente di un uso fazioso degli strumenti di garanzia della legalità costituzionale (come è accaduto di recente nel caso dell'impeachment della Presidente Dilma Rousseff). ${ }^{5}$

Le contraddizioni che si costituiscono attraverso e all'interno del processo di recezione delle idee occidentali rappresentano un tratto distintivo dello sviluppo di molti paesi che hanno intrapreso il cammino dell'indipendenza dopo un periodo di colonizzazione. Sul versante degli studi giuridici, ad esempio, si possono incontrare diversi rilievi critici delle più recenti esperienze di cooperazione internazionale, finalizzate a coniugare investimenti finanziari nei paesi in via di sviluppo con la promozione e la tutela dei diritti fondamentali. ${ }^{6}$

Il sentimento di disillusione verso la realizzazione delle istanze di emancipazione individuale e sociale è stato interpretato, in diverse epoche e contesti, da molti scrittori degli "ex-territori coloniali." È significativo, inoltre, il fatto che questi ultimi hanno più o meno apertamente "risposto" alla tradizione letteraria occidentale opponendo una autonoma ricerca di stili e di metodologie. ${ }^{8}$ Un elemento che accomuna la prosa degli scrittori sudamericani e africani, ad esempio, è l'esaltazione del contrasto tra le proclamazioni messianiche di libertà delle costituzioni post-coloniali e la continuità dell'ordine sociale del colonialismo. ${ }^{9}$ In tal senso, la carica emotiva degli autori che avevano accompagnato la fase indipendentista si spegne negli anni successivi, lasciando spazio al pessimismo della ragione. ${ }^{10}$

\section{MACHADO DE ASSIS E LA SOCIETÀ BRASILIANA TRA IMPERO E REPUBBLICA}

La complessità delle relazioni di potere è ascrivibile a molte opere di Machado de Assis, che è tuttora considerato uno dei maggiori esponenti della letteratura brasiliana. Attraverso i suoi scritti si accede ad un ritratto della società carioca in un'epoca in cui lo Stato brasiliano era ancora alla ricerca di un definitivo assetto istituzionale. La narrativa machadiana, in questo senso, 
rappresenta una testimonianza diretta degli anni che segnarono il tramonto della monarchia costituzionale ${ }^{11}$ e la nascita della Repubblica presidenziale ispirata all'esperienza del liberalismo nordamericano.

Machado de Assis è un osservatore importante perché racconta il suo paese sotto una prospettiva diversa rispetto ai suoi contemporanei, soprattutto a partire da quella che è comunemente definita «la seconda fase dell'opera machadiana». ${ }^{12}$ Mentre, da un lato, chiede di essere considerato «un uomo del suo tempo e del suo paese», dall'altro, lo scrittore rivendica la libertà di trarre ispirazione da «temi remoti e lontani». Così, nella sua fase più matura, la prosa di Machado de Assis percorre un orizzonte letterario del tutto inedito, collocandosi in aperta polemica con diversi scrittori del periodo imperiale che, seguendo la tradizione, consideravano "autenticamente brasiliano" solamente il romanzo ispirato dalla realtà locale. ${ }^{13}$

Questo spirito di rottura si esprime pienamente in Memórias Póstumas de Brás Cubas ${ }^{14}$, nel quale l'autore si avvale di molte risorse letterarie e filosofiche della tradizione europea per esporre la sua percezione della società nazionale in un ottica non più limitata dall'esperienza positiva dei fatti. L'opera è stata accolta dalla critica come il primo romanzo "realista" della letteratura brasiliana, nonostante sia solamente in parte riconducibile a questo approccio. Oltre alla narrazione delle vicende di vita e delle condizioni delle classi sociali dell'epoca, infatti, assumono una rilevanza fondamentale le componenti del romanticismo europeo e del romanzo di fantasia, a cui l'autore fa ampio rinvio aggiungendo singolari sfumature di sarcasmo.

La rivoluzione di Machado consiste dunque nell'aver reintepretato i temi e i personaggi dei romanzi europei da una prospettiva autenticamente brasiliana, dando vita ad un scrittura del tutto originale nel suo stile e presentando una realtà locale sconosciuta agli occhi del mondo. L'ambiente esotico, l'indio coraggioso e selvaggio, il colono avventuriero lasciano il posto al ritratto di una società arcaica, in cui, dietro gli abiti e i costumi antichi, si nasconde la violenza di una civilizzazione fondata sullo schiavismo e sul predominio della razza bianca. ${ }^{15}$

La diffusione dell'opera di Machado de Assis in Europa avviene già venti anni dopo la sua morte, in un periodo in cui la critica letteraria del Vecchio Continente era poco incline ad aperture verso orizzonti culturali così remoti.

Nel 1928 viene pubblicata la traduzione in italiano di «Memórias Póstumas de Brás Cubas», presentata nell'introduzione di Mario da Silva come «il più originale e umano romanzo che possegga la letteratura brasiliana e uno dei più profondi di tutta la letteratura della lingua portoghese». ${ }^{16}$ È opportuno ricordare, tuttavia, che diversi anni prima era già uscito in Francia un ampio studio su Machado de Assis condotto da Victor Orban, Anatole France e Oliveira Lima: ${ }^{17}$ un omaggio a «le genié latin» la cui opera artistica ha dato prova di un approccio narrativo, al contempo, «nazionale ed universale». ${ }^{18}$ 
Negli anni a seguire tale interpretazione dello stile machadiano non perde la sua attualità e viene ribadita nella riedizione italiana del romanzo, pubblicata nel 1953, con il titolo «Memorie dall'aldilà». Nelle pagine dell'introduzione Laura Marchiori, che è anche la traduttrice dell'opera, definisce Machado de Assis «uno degli scrittori più universali del suo tempo», capace di condurre uno studio approfondito dell'animo umano e dei conflitti psicologici restando immune da qualsiasi «interferenza del folklore». ${ }^{19}$ Questa presentazione è capace cogliere un tratto fondamentale, il quale trapela dal pessimismo di fondo che caratterizza la maggior parte degli scritti di Machado e dall'ironia più esplicita con cui quest'ultimo dipinge le gesta, del tutto prive di audacia, dei suoi personaggi. La trama del romanzo, articolata attorno alle vicende della vita di Brás Cubas, si interrompe infatti più volte per lasciare spazio alla rappresentazione dei "residui" di un Brasile precapitalista: lo schiavismo, la classe parassitaria e arrogante dei ricchi proprietari, la staticità del tessuto sociale, il conservatorismo dei costumi.

La posizione di Machado de Assis acquista in questo senso un duplice rilievo per lo studioso europeo: da un lato, costituisce una testimonianza delle tensioni sviluppatesi intorno ai processi di diffusione oltreoceano del pensiero politico e filosofico del Vecchio Continente, dall'altro, identifica la realtà brasiliana come il prodotto di questa contraddizione tra l'arcaico e il moderno, tra i canoni antichi e il progresso guidato dell'ambizione individuale. La terra del protagonista, lontana dall'essere la patria romantica dell'eroe borghese europeo, è il luogo di una oscena polarizzazione sociale. ${ }^{20}$

\section{UN ROMANZO "DI ROTTURA”"1}

La scena del racconto è la capitale carioca, la Rio de Janeiro che in quel periodo era probabilmente la più europea delle città del Nuovo Continente. Il trasferimento della Corona portoghese tra il 1808 e il 1821 aveva infatti condizionato profondamente la crescita della città, che a partire da questo momento diventa un punto di passaggio di molti studiosi e diplomatici d'oltreoceano. ${ }^{22}$ Rio de Janeiro è il cuore dell'ex-colonia sudamericana nel XIX secolo, sebbene non l'unico: ${ }^{23}$ i grandi centri di produzione di caffè, San Paolo e Minas Gerais, incalzano infatti il primato economico carioca per tutto il Secondo Impero (1840-1889) e diventano i poli di riferimento della vita politica durante la Prima Repubblica (1889-1930). ${ }^{24}$

La voce della narrazione è quella di Brás Cubas, che parla dall'oltretomba. È infatti morto di polmonite e, soltanto quando è passato a miglior vita, si decide di scrivere la sua autobiografia. L'incipit del romanzo preannuncia la fusione degli stili del racconto: il sarcasmo coinvolge da subito il lettore, verso il quale Brás Cubas alterna un tono ossequioso e sferzate di umorismo molto vicine all'affronto; ${ }^{25}$ l'introspezione psicologica mostra l'animo sconfitto, ma non affranto, del protagonista; la componente di fantasia fa la sua apparizione con la descrizione della veglia funebre da parte dello stesso «defunto-autore». Il carattere irriverente del romanzo trova il suo apice in un passaggio dell'apertura. 
Brás Cubas si addentra in una comparazione storica per "giustificare" le sue memorie postume, spiega perciò che non è la prima volta in cui un defunto sia anche uno scrittore: si riconduce infatti alla tradizione biblica, in particolare a Mosè, il quale descrisse la sua morte nel Libro dell'Esodo. Tuttavia, precisa il protagonista, la «differenza radicale» tra il suo libro e la Sacra Scrittura consiste nel fatto che Mosè raccontò la sua morte alla fine della storia e non all'inizio. Infine, la scelta stilistica di Bras Cubas è rappresentata dal rifiuto di una «usanza volgare», ossia iniziare le memorie dai primi anni di vita, e dalla preferenza per una «scrittura più galante e più moderna».

La veglia funebre è accompagnata dal pianto di Virgilia, l'amore nascosto del defunto-autore, morto celibe e con soli undici amici che presenziano al suo funerale. Un'ironia macabra trapela fin dalle prime pagine del libro e rimane fino alla fine sullo sfondo del racconto. La "narrazione-da-morto" permette infatti a Brás Cubas di riportare i fatti della sua vita con una totale esenzione, il trapasso rappresenta una liberazione dal "peso dell'esistenza" più volte rievocato nel racconto degli episodi della vita. ${ }^{26}$

Il malessere interiore del defunto-protagonista viene posto immediatamente a contrasto con il benessere economico di cui ha sempre goduto in vita.

Brás Cubas è il simbolo della élite carioca del XIX secolo, una classe di renters, proprietari di terre e di schiavi, senz'altro estranei all'etica individualista del self-made man che aveva caratterizzato l'ascesa della borghesia europea. La sua educazione è fortemente influenzata dalla famiglia schiavista: durante l'infanzia il gioco preferito è cavalcare Prudêncio, figlio coetaneo di uno dei servitori della famiglia. Così, si guadagna l'appellativo di «menino diabo» tra la servitù costretta a sottomettersi ai suoi vizi per evitare le violente sfuriate, come quando colpisce in testa una schiava che gli rifiuta il dolce di cocco.

Nel racconto dell'infanzia viene anticipato l'elemento che secondo diverse ricostruzioni costituisce il filo conduttore dell'opera, ossia il "capriccio." Nessuno dei personaggi di primo piano del romanzo è animato infatti da nobili sentimenti: al contrario, inseguono l'ascesa sociale o la carriera politica per consolidare il potere della famiglia di appartenenza o per la semplice soddisfazione personale. Sotto questa prospettiva il capriccio rappresenta una declinazione in chiave satirica dei sogni e delle ambizioni degli eroi del romanticismo europeo, di cui Brás Cubas e gli altri ne sono evidentemente la "versione goffa".

Il capriccio è infine all'origine dei tormenti dell'animo dei personaggi che conducono delle esistenze quasi del tutto prive di iniziative nobili e incapaci di conquistare le emozioni del lettore. Così, ad esempio, l'incapacità di resistere a Marcela e i suoi "costosi capricci" condannano il giovane Brás Cubas a subire la punizione del padre, il quale, quando arriva il conto della gioielleria, deciderà di imbarcare a forza il figlio in una nave diretta in Portogallo, per iniziare gli studi di diritto all'Università di Coimbra. Una volta tornato in terra natia, però, è Brás Cubas la vittima dei fallimentari progetti del padre, che a tutti i costi vuole 
vedere suo figlio seduto alla Camera dei Deputati. Al fine di garantire il seggio al giovane candidato, l'uomo aveva infatti programmato il matrimonio dell'ignaro figlio con Virgilia, appartenente all'importante discendenza dei Dutra. Il disegno tuttavia fallisce proprio quando Bras Cubas, dapprima disinteressato alla proposta del padre, incomincia a provare un forte sentimento per la donna che avrebbe dovuto essere la sua futura sposa. Il padre di Virgilia infatti preferisce cedere la mano della figlia a Lobo Neves, anche lui intenzionato a candidarsi alla Camera dei Deputati. Infine, il capriccio di incontrare di nuovo Virgilia dà avvio ad una relazione clandestina tra il protagonista e la donna, che tuttavia è destinata a non durare a lungo. Poco tempo dopo Virgilia è infatti costretta a lasciare Rio de Janeiro al seguito del marito Lobo Neves, il quale è stato eletto presidente di una provincia del Nord del Brasile.

La narrazione prosegue tra progressi e delusioni: Cubas diventa deputato, ma non riesce a divenire ministro e i suoi progetti di fondare un giornale di opposizione svaniscono. Si sposa con una giovane donna presentatagli dalla sorella, che però poco tempo dopo muore e lo lascia vedovo.

Infine, ritorna l'elemento del fantastico nella narrazione: Brás Cubas tenta di redimere la sua inutile esistenza cercando di fare qualcosa che lo riesca ad «elevare tra le prime file degli esseri umani, al di sopra della scienza e della ricchezza, perché diretta ispirazione divina». È l'«Emplasto Bras Cubas», una panacea contro tutti i mali. Ironia della sorte, però, si ammala di polmonite per essere uscito in strada nel tentativo di prendersi cura del suo progetto («il caso ha determinato il contrario, e per questo ora voi soffrite di ipocondria»).

L'epilogo del romanzo, al di là dell'ironia, non lascia alcuno spazio alla realizzazione dei progetti di vita di Brás Cubas e degli altri personaggi. Il defunto-autore, nonostante tutto, si rallegra dell'assenza di ulteriori patimenti, ossia per non aver mai dovuto pagare il pane con il sudore della sua fronte e per non essere impazzito come il suo amico Quincas Borba. E in ultimo, aggiunge: «non ho avuto figli, non ho trasmesso a nessuna creatura l'eredità della nostra miseria». ${ }^{28}$ In tal senso si spiega la singolare dedica collocata in epigrafe alle memorie: «al verme che per primo ha rosicchiato la carne fredda del mio cadavere».

\section{LA PIAGA DELLO SCHIAVISMO}

Non è sufficiente una sola interpretazione per comprendere l'opera nella sua densità di stili e contenuto. Le diverse posizioni che si sono confrontate nella critica (non solo letteraria) lasciano intendere la difficoltà di trovare una collocazione definitiva del romanzo secondo gli schemi classici della narrativa. Memórias Póstumas de Brás Cubas è, tuttavia, un opera che non vuole fare "solo" narrativa, pertanto, una delle chiavi di lettura che si impone è quella della polemica nell'ambito della quale l'autore esprime la sua idea di «istinto di nazionalità». ${ }^{29}$

Machado de Assis, come già detto, è uno scrittore critico verso gli esponenti della letteratura nazionale a lui contemporanei, di cui non condivide 
l'ideologia "localista": ritiene infatti che rifiutando il confronto con i temi europei si sarebbe ritardato lo sviluppo della narrativa brasiliana. ${ }^{30}$ Nella seconda fase della prosa di Machado de Assis la contemplazione della natura, il racconto dei conflitti passionali e dei costumi dei popoli passano in secondo piano. È invece la coscienza di un popolo, le sue miserie e la sua falsa moralità che l'autore voler esplorare e portare alla luce, inaugurando un cammino letterario all'epoca del tutto nuovo. Machado de Assis è consapevole che una scrittura apertamente filosofica o politica non avrebbe diffusione tra i suoi connazionali, non esistendo una tradizione di opere basata su «studi solidi». ${ }^{31}$ La sua intenzione è quella di arrivare alla sua gente, ed è per questo che sceglie il romanzo e fa ampiamente ricorso a tutte le risorse stilistiche a disposizione per raggiungere il suo obiettivo.

Uno degli strumenti più efficaci della prosa machadiana è la costruzione dei personaggi, attraverso la quale è possibile percepire tutta la sfiducia dell'autore sia verso la società brasiliana, sia verso la natura umana. ${ }^{32}$ L'obiettivo della critica di Machado de Assis è specialmente l'élite della Capitale: ciò appare evidente nella misura in cui viene enfatizzata la fiacchezza morale ed esistenziale di Brás Cubas e dei suoi pari, arricchita dalla descrizione sarcastica delle loro vite e dei loro insuccessi. ${ }^{33}$ Nondimeno, è opportuno tener presente che il punto di osservazione dal quale Machado d'Assis racconta la società del Secondo Impero brasiliano è condizionato dalla sua singolarissima esperienza esistenziale.

La vita dello scrittore è infatti caratterizzata da una irrefrenabile ascesa professionale, che gli ha permesso di attraversare gradualmente la società brasiliana dal basso verso l'alto. Machado d'Assis cresce nel barrio Livramento, proveniente da una famiglia discendente da schiavi "alforriados", ossia liberati da un precedente proprietario. Per tutta la sua infanzia e la sua adolescenza sopravvive in condizioni precarie, ma non miserabili: fa parte di una "famiglia aggregata", ossia dipendente di una altra famiglia di ricchi proprietari di terre che dava lavoro ai genitori. Conduce degli studi discontinui, che però risultano sufficienti a farlo assumere presso la tipografia di Paula Britto, frequentata da diversi autori importanti. Poco dopo la pubblicazione del suo primo poema nel $1855^{34}$ e la relativa stabilità economica, passa dalla mansione di tipografo a quella di revisore e, infine, alla redazione del "Correio Mercantil". A trenta anni è già uno scrittore conosciuto.

Da figlio di imbianchino e di una lavandaia Machado d'Assis diventa dapprima giornalista, successivamente poeta, drammaturgo, scrittore di romanzi e, infine, Presidente Onorario dell'Academia Brasileira de Letras.

La particolare sensibilità verso i temi della disuguaglianza razziale, unita alla disillusione verso i proclami liberali della borghesia brasiliana, sono il prodotto sia di esperienze di vita privata sia del contatto diretto con la politica.

Sotto il primo profilo, si noti che la storia di Machado offre, di per sé, una testimonianza chiara della crudeltà sprigionata da un sistema di apartheid e delle consuetudini discriminatorie in materia miscegenation. Nel 1869 nono- 
stante lo schiavismo sia stato profondamente criticato dalla corrente liberale, il Parlamento brasiliano ancora non ha raggiunto un accordo sull'abolizione della antica piaga. ${ }^{35} \mathrm{E}$ in questo anno che Machado de Assis, il cui colore della pelle rivelava le discendenze africane del nonno paterno, affronta per l'ennesima volta il preconcetto razziale, questa volta da parte della famiglia portoghese della sua futura moglie Carolina. ${ }^{36} \mathrm{Il}$ fratello, Faustino Xavier, si oppone infatti al matrimonio, ma alla fine lo scrittore riesce a vincere le resistenze grazie alla sue conoscenze illustri.

Sul versante politico, invece, è importante ricordare che nel 1874 Machado entra nel Ministero dell'Agricoltura, dove lavora in una sezione incaricata di vigilare l'applicazione di uno dei più importanti provvedimenti del parlamento brasiliano a favore della fine della schiavitù: la "Lei do ventre livre". La legge fu promulgata il 28 settembre 1871 e stabiliva che a partire da quella data tutti i figli nati da una donna in condizioni di schiavitù dovevano considerarsi liberi, mentre i genitori continuavano a rimanere sottomessi ai loro proprietari. Dopo anni di accese discussioni alimentate dalle pressioni diplomatiche, soprattutto inglesi, viene data alla luce una riforma molto contestata e che, soprattutto, non produce rilevanti effetti nell'immediato. Sotto il primo profilo, si ricorda che il processo di approvazione della legge aveva dovuto superare la dura opposizione dei parlamentari eletti negli stati di Minas Gerais, San Paolo e Rio de Janeiro, dove i profitti delle élite dei coltivatori di caffè ancora dipendevano ampiamente dalla manodopera servile. D'altra parte, l'attuazione del provvedimento svuotava di senso i principi ispiratori della riforma: $\mathrm{i}$ «figli liberi» di genitori ancora in condizioni di schiavitù dovevano rimanere in custodia presso i «signori» fino all'età di 21 anni (prevedendo la possibilità per i tutori di avvalersi del loro lavoro) o, alternativamente, potevano essere affidati allo Stato (con la conseguente nascita del diritto del signore ad essere indennizzato per la perdita delle prestazioni del minore). ${ }^{37}$

Questa riforma era evidentemente ben lontana dall'esprimere l'idea di liberalismo patrocinata dagli autori europei e, probabilmente, per Machado de Assis è l'ennesima dimostrazione del provincialismo del paese latinoamericano. Nel percorso abolizionista del Brasile non vi è infatti alcuna traccia di una genuina maturazione di istanze egualitarie. Così come per molti altri paesi del Continente Americano, la rottura con la tradizione schiavista viene forzata dai parlamenti europei, la cui retorica dei diritti e dell'autonomia dell'individuo cresceva di pari passo con l'avanzamento del processo di industrializzazione. Ciò spiega perché l'Inghilterra in quel periodo conduceva le pressioni diplomatiche verso molti degli ex-mercati coloniali, compreso il Brasile.

È importante ricordare, infatti, che nel 1845 il Parlamento britannico aveva approvato un provvedimento destinato a inasprire notevolmente i rapporti con l'Impero del Brasile, il c.d. Aberdeen Act, che deve il nome all'allora Segretario degli Esteri della Corona inglese. In realtà si tratta di un dispositivo rivolto a dare effettività ad un accordo di un ventennio precedente siglato dal 
Re inglese Giorgio V e dell'Imperatore del Brasile Dom Pedro I: ${ }^{38}$ la necessità di un rafforzamento delle misure di contrasto al commercio degli schiavi si era resa necessaria alla luce dell'accondiscendenza, nemmeno troppo velata, riservata dalle istituzioni brasiliane verso i trafficanti. ${ }^{39}$ Pertanto, con un'iniziativa unilaterale, il Parlamento britannico ripristinava la giurisdizione dei tribunali marittimi inglesi per la repressione del traffico di schiavi in alto mare, precedentemente sospesa in attuazione della convenzione che affidava tale compito alle commissioni giudicanti "miste", ossia composte da arbitri brasiliani e britannici. Infine, veniva conferito alla marina reale la prerogativa di intercettare in alto mare le navi brasiliane sospettate di nascondere e trasportare degli schiavi, catturarne eventualmente $i$ trafficanti per condurli in giudizio davanti ad un tribunale marittimo inglese («Her Majesty's High Court of Admiralty and any Court of Vice Admiralty within Her Majesty's Dominions »).

La storiografia ci segnala, tuttavia, che l'attuazione del provvedimento non impedì la continuazione clandestina della tratta. ${ }^{40}$ L'unico effetto immediato fu quello di aumentare il costo degli schiavi nel mercato brasiliano, dato il maggiore rischio che i trafficanti dovevano affrontare nell'attraversare l'Atlantico. Un simile risvolto della vicenda fu reso possibile dalla già accennata ambiguità della classe dirigente delle ex-colonie sudamericane, i cui privilegi sociali e politici poggiavano sui proventi della tratta degli africani.

La formazione dello Stato brasiliano assumeva pertanto due opposte inclinazioni: da un lato, la ricerca di una legittimazione internazionale promuoveva l'adozione formale dei principi liberali nella Costituzione imperiale del $1824,{ }^{41}$ dall'altro, l'assenza di una politica abolizionista rivelava l'incapacità delle istituzioni di rimediare all'incoerenza etica della élite che rivendica una "fratellanza ideale" con la borghesia europea. L'esportazione del liberalismo nel Nuovo Continente si risolve, almeno in un primo momento, nella nascita di democrazie censitarie e su base razziale che segneranno la storia della "civilizzazione" d'America dal Nord al Sud. ${ }^{42}$

In un secondo momento prevalsero, tuttavia, le ragioni dell'abolizionismo, nella misura in cui la transizione alla manodopera salariata risultava essenziale per l'attrazione di investimenti stranieri e per aprire un nuovo mercato ai prodotti della crescente industria inglese. Dopo la Lei do Ventre Livre è infatti la Lei Aurea ("la legge d'oro") del 1888 che abolisce ufficialmente la schiavitù.

Durante il suo incarico al Ministero dell'Agricoltura Machado d'Assis osserva quest'ultimo momento di vigenza dello schiavismo senza credere minimamente in una liberazione compiuta attraverso il diritto positivo. È facile rilevare il generale scetticismo dell'autore verso le transizioni storiche a cui avrà modo di assistere durante la sua vita: la stessa proclamazione della Repubblica, la quale appare nelle opere Esaú e Jaco e Memorial de Aires, è presentata come una cambiamento di facciata, dove l'etica e la base ideologica delle classi dirigenti rimangono invariate. ${ }^{43}$ 


\section{UNA «MODERNIZZAZIONE CONSERVATRICE»}

Memórias Póstumas de Brás Cubas è infine un romanzo sulle classi sociali che caratterizzarono la formazione dello Stato brasiliano, le ambizioni che le accomunarono e il diverso destino che l'ordine sociale del Secondo Impero riservò a ciascuna di esse. Sotto questa prospettiva, il pessimismo machadiano che fa da sfondo alla narrazione esprime una sfiducia verso la bontà dei sentimenti umani, la capacità dell'uomo di liberarsi dall'invidia (trasformata in virtù dalla strampalata filosofia "humanitista" di Quincas Borba), ${ }^{44}$ l'effettiva esistenza di ciò che viene chiamato "progresso".

Il distacco riservato da Machado de Assis nei confronti dei suoi contemporanei è "totale", molto simile a quello di Brás Cubas, al quale la morte ha donato il privilegio di narrare i fatti della sua vita con leggerezza e ironia. Nessuna delle classi sociali viene risparmiata, nemmeno i poveri ostentano delle virtù o una coscienza morale superiore a quella dei proprietari. Si riportano, in tal senso, due importanti passaggi dell'opera, nei quali vengono narrati altrettanti episodi che intendono esprimere, secondo la più attenta critica letteraria, l'idea machadiana della «sorte dei poveri». ${ }^{45} \mathrm{~A}$ metà del romanzo (Capitolo LXVIII) appare di nuovo Prudêncio, il quale, dopo essere stato liberato dal padre di Bras, aveva usato i suoi risparmi per comprare a sua volta uno schiavo a cui non risparmia le frustate che aveva ricevuto dai Cubas. Poche pagine dopo, viene introdotta la figura di Dona Plácida, vedova con una figlia denutrita a carico, costretta ad umili lavori giorno e notte che non riescono comunque a garantire ad entrambe un esistenza dignitosa. La donna si mostra in un primo momento riluttante rispetto alla proposta di offrire rifugio, nella sua piccola casa, agli incontri segreti tra il protagonista e Virgilia (già sposa di Lobo Neves). Successivamente Bras Cubas, con un'impennata di cinismo, racconta come le perplessità di Placida scomparvero davanti all'offerta di una discreta somma di denaro, accompagnata da discorsi romantici sul suo amore per Virgilia (Capitolo LXX).

Dalle pagine di Memórias Póstumas de Brás Cubas si innalza una critica che dunque sarebbe riduttivo limitare alla sola élite brasiliana. La mentalità schiavista, il destino dei poveri, che nonostante una dura vita di lavoro devono continuare a cercare protezione presso i "signori proprietari", rappresentano un capitolo di un racconto molto più lungo: quello della sconfitta della cosiddetta "civilizzazione" del Brasile e delle mistificazioni del pensiero positivista che l'ha guidata. Si presti attenzione all'ironia con la quale l'autore sminuisce gli studi di diritto del giovane Brás Cubas: «Avevo conquistato a Coimbra la nomea di gran festaiolo; ero un accademico dissoluto, superficiale, tumultuoso e petulante, completamente donato alle avventure, facendo del romanticismo pratico e del liberalismo teorico, vivendo di fede pura negli occhi neri e nelle costituzioni scritte». Ma il positivismo, creazione di una Europa attraversata dalla paura delle insurrezioni, se da un lato assume il linguaggio dei rivoluzionari nelle carte costituzionali che "pianificano" una società di uguali, dall'altro, con le sue verità scientifiche, contribuisce a smentire le utopie di uguaglianza dimostrando la 
"gerarchia delle razze" che legittima il colonialismo. ${ }^{46}$

La sfiducia di Machado d'Assis verso le capacità della élite brasiliana di aprire la strada allo sviluppo delle istituzioni liberali del Brasile ha avuto, in un certo senso, un ruolo premonitore rispetto alle successive fasi della storia del paese. Il ritardo del Brasile nel processo di industrializzazione, così come le fasi alterne che ha vissuto il consolidamento della democrazia, sono in gran parte imputabili alla forma oligopolistica che per lungo tempo hanno assunto i vari settori dell'economia ed ai pericolosi legami che questi ultimi, in alcuni frangenti storici, hanno sviluppato con i settori meno liberali delle forze armate.

Oggi la cultura politica e la struttura della società brasiliana è certamente distante da quella narrata dal romanzo di Machado de Assis. Tuttavia, ancora una volta, il processo di modernizzazione che ha attraversato il Brasile negli ultimi anni non è ascrivibile ad un cambiamento culturale guidato della élite, bensì ad un massiccio interventismo statale nell'economia, nell'educazione e, in generale, nell'accesso ai diritti sociali. Sotto questo profilo la storia brasiliana presenta dei punti in comune con quella italiana. ${ }^{47}$

Sta accadendo però qualcosa di nuovo, come testimoniano le tensioni che oramai da due anni attraversano le istituzioni e la popolazione brasiliana: ${ }^{48}$ apparentemente questa presenza dello Stato è divenuta ingombrante. Improvvisamente compaiono richieste di maggiore libertà per le imprese, di abbassamento della pressione fiscale, di flessibilizzazione della disciplina dei rapporti di lavoro e, infine, di riduzione della spesa pubblica. Ecco un secondo paragone possibile con l'Italia del "dopo-tangentopoli": l'Italia che chiede meno politica, quando non la rifiuta esplicitamente perché preferisce l'economia. Non è agevole dar conto in poche righe dei molteplici punti di osservazione da cui si potrebbe tracciare un parallelo tra le inchieste Lava-Jato e Mani Pulite. E preferibile, in questa sede, limitarsi a rilevare la pressione mediatica che accompagna le indagini del potere giudiziario, il crescente distacco dei cittadini dal sistema dei partiti e la riduzione del dibattito politico a due opposti schieramenti di "favorevoli" e "contrari" all'opera della magistratura.

Mentre tutto questo accade sullo sfondo del ritratto del Brasile del XXI secolo appare una "nuova classe" di grandi proprietari, alla maniera di Bras Cubas, sebbene sicuramente meno identificabili perché celati dietro le sigle delle corporations che operano nella finanza e nei media. Sono questi proprietari che oggi dettano i tempi e le dinamiche della politica istituzionale e che chiedono un cambiamento di rotta difficilmente riconducibile nei confini dell'esperienza costituzionale del Brasile democratico. 


\section{RIFERIMENTI}

ALBERTAZZI, Silvia. Lo sguardo dell'altro. Le letterature postcoloniali. Roma: Carocci, 2000.

AMIN, Samir. L'eurocentrisme, critique d'une idéologie. Parigi: Anthropos, 1988.

ASHCROFT, Bill; GRIFFITHS, Gareth; TIFFIN, Helen. The Empire Writes Back: theory and practice in post-colonial literatures. London-New York: Routledge, 2002.

CALMON, Pedro. História social do Brasil. São Paulo: Martins Fontes, 2002. v. 3.

CANOTILHO, José Joaquim Gomes. Constituição-dirigente e vinculação do legislador: contributo para a compreensão das normas constitucionais programáticas. Coimbra: Coimbra Editora, 1982.

CORONEL, Cristina et al. Usaid en Paraguay: la asistencia como estrategia de dominación. Asunción: Base Investigaciones Sociales, 2012.

COUTINHO, Jacinto Nelson de Miranda; AMATUZZI, Bruna Araujo. Ensaio sobre a constitucionalidade, a razoabilidade e a prisão especial no Processo Penal contemporâneo. Disponível em: < http://emporiododireito.com.br/ ensaio-sobre-a-constitucionalidade-a-razoabilidade-e-a-prisao-especial-no-processo-penal-contemporaneo-por-jacinto-nelson-de-miranda-coutinho-e-bruna-araujo-amatuzzi/>. Acesso em: 27 jul. 2016.

FANON, Frantz. Black Skin, White Masks. New York: Grove Press, 1967.

FRANCE, Anatole; ORBAN, Victor; LIMA, Oliveira. Machado de Assis et son oeuvre littéraire. Parigi: Louis-Michaud, 1909.

GUITIAN, Manuel. Fund conditionality: evolution of principles and practices. Washington: IMF Pamphlet Series, 1981.

JINKINGS, Ivana; DORIA, Kim; CLETO, Murilo. Por que gritamos ao golpe? São Paulo: Boitempo Editorial, 2016.

LAJOLO, Marisa. Machado de Assis: literatura comentada. São Paulo: Abril Cultural, 1980.

LAVER, Roberto. The World Bank and Judicial Reform: Overcoming "Blind Spots" in the Approach to "Judicial Independence." Duke Journal of Comparative and International Law, Durham, v. 22, p. 183-238, 2012.

LAZARUS, Neil. Resistance in Post-Colonial African Fiction. New York: Yale University Press, 1990.

LOSURDO, Domenico. Controstoria del liberalismo. Roma-Bari: Laterza, 2005. 
MACHADO DE ASSIS, Joaquim Maria. Críticas. Joinville (SC): Clube de Autores, 2010.

. Memorias póstumas de Brás Cubas. São Paulo: Metalibri, 2006.

. Memorie dall'aldilà. Milano: Rizzoli, 1953.

. Memorie Postume di Braz Cubas. Milano: Edizioni Cobbaccio, 1928.

. Obra completa. Rio de Janeiro: Nova Aguilar, 1994. v. 3.

MALAGUTI BATISTA, Vera. O positivismo como cultura. Passagens Revista Internacional de História Política e Cultura Jurídica, Rio de Janeiro, v. 8, n. 2, p. 293-307, maio/ago. 2016.

MATTEI, Ugo; NADER, Laura. Il saccheggio. Regime di legalità e trasformazioni globali. Milano-Torino: Bruno Mondadori, 2010.

MEMMI, Albert. The Colonizer and the Colonized. Boston: Beacon Press, 1965.

MOTTOSO, Kátia de Queirós. Modèles politiques et culturels au Brésil: emprunts, adaptations, rejets, XIXe et XXe siècles: colloque de l'Institut de Recherches sur les Civilisations de l'Occident Moderne, les 8, 9 et 10 mars 1999 en Sorbonne. Parigi: Presses de l'Univ. de Paris-Sorbonne, 2003.

PINHO, Rodrigo César Rebello. Da organização do estado, dos poderes e histórico das constituições. São Paulo: Saraiva, 2001.

POLASKI, Sandra. Protecting Labor Rights through Trade Agreements: an Analytical Guide. Journal of International Law and Policy, n. 14, p. 13-26, July 2004.

SCHWARTZ, Roberto. Que horas são? São Paulo: Companhia das Letras, 1987.

SEN, Amartya. La democrazia degli altri: perché la libertà non è un invenzione dell'Occidente. Milano: Oscar Mondadori, 2010.

SILVA SANTOS, Valdoir de. Multiculturalismo e pluralismo jurídico: a luta pela liberdade e o direito. São Paulo: Biblioteca24horas, 2013.

SOMMA, Alberto. Introduzione al Diritto Comparato. Roma-Bari: Laterza, 2014.

STAFFEN, Márcio Ricardo. Hegemonia e direito transnacional? Revista Novos Estudos Jurídicos, Itajaí (SC), v. 20, n. 3, p. 1166-1187, set./dez. 2015.

TAVARES, Juarez; PRADO, Geraldo. O Direito Penal e o Processo Penal no Estado de Direito: analise de casos. Florianópolis: Empório do Direito, 2016.

VERISSIMO, José. História da Literatura Brasileira. Brasília: Ministério da Cultura-Fundação Biblioteca Nacional, 1915.

XYPOLIA, Ilia. Eurocentrism and Orientalism. In: RAY, Sangeeta et al. The Encyclopedia of Postcolonial Studies. New Jersey: Wiley-Blackwell, 2016. 
1 STAFFEN, 2015.

2 Su questo tema, tra i vari contributi, vedere: XYPOLIA, 2016; SEN, 2010; AMIN, 1988.

3 Tra i più recenti contributi della dottrina comparatistica italiana in tema di circolazione delle idee occidentali nel contesto latinoamericano, cfr. SOMMA, 2014, p. 119: "Giungiamo così a lambire il tema della modernità come vicenda che occorre analizzare al plurale, per individuare più centri da cui essa prende corpo, magari proprio laddove l'impostazione tradizionale pretende di ravvisare una semplice periferia. [...] In tale prospettiva la diffusione della modernità, che alcuni reputano inevitabile, cessa di coincidere con l'occidentalizzazione dei contesti in cui si manifesta. Contesti nei quali non si verificano meri recepimenti, bensì, quantomeno, contaminazioni tra influenze esterne comunque esercitate e dati identitari interni in perenne trasformazione, ma ineliminabili».

4 SILVA SANTOS, 2013.

5 Per una analisi critica in merito alla possibilità di rilevare «crimini di responsabilità» nel corso del mandato di Dilma Rousseff (necessari, ai sensi dell'art. 86 della Costituzione brasiliana, per la procedibilità dell'impeachment), cfr. TAVARES; PRADO, 2016. Per una critica rivolta, più in generale, alle ambiguità del processo di impeachment, cfr. JINKINGS; DORIA; CLETO, 2016.

6 Cfr. GUITIAN, 1981; LAVER, 2012; POLASKI, 2004; CORONEL et al., 2012. Per una analisi critica più generale degli effetti della condizionalità dei trattati internazionali di investimento, cfr. MATTEI; NADER, 2010.

7 MEMMI, 1965; FANON, 1967. Sul versante della critica letteraria brasiliana, in questa prospettiva, cfr. SCHWARTZ, 1987.

8 ASHCROFT; GRIFFITHS; TIFFIN, 2002. Tra le ricostruzioni della letteratura postcoloniale operate dagli studiosi italiani, cfr. ALBERTAZZI, 2000.

9 Su questo punto cfr. LAZARUS, 1990.

10 Il "messianismo" dei rivoluzionari indipendentisti viene riprodotto infatti in molte opere africane, ma la sua celebrazione critica in contrapposizione alla miseria della realtà popolare è stata resa popolare dall'opera di FANNON, 1969. Cfr., inoltre, LAZARUS, 1990, p. 27.

11 Con riferimento al "quasi-parlamentarismo" che caratterizzò il Secondo Impero del Brasile cfr. PINHO, 2001, p. 144-145: «Não se tratava de um regime parlamentarista puro, pois, para se conservar no poder, o Gabinete precisava continuar a merecer a confiança do Parlamento e também do Imperador, titular do Poder Moderador. Dom Pedro II utilizou-se disso com sabedoria para alternar no exercício do poder liberais e conservadores, conforme as conveniências de cada momento».

12 Su tutti, cfr. VERISSIMO, 1915, p. 188.

13 MACHADO DE ASSIS, 1994.

14 MACHADO DE ASSIS, 2006.

15 LAJOLO, 1980, p. 102.

16 MACHADO DE ASSIS, 1928, p. 7.

17 FRANCE; ORBAN; LIMA, 1909.

18 MOTTOSO, 2003, p. 37.

19 MACHADO DE ASSIS, 1953, p. 5.

20 SCHWARZ, 2000, p. 9.

21 VERISSIMO, op. cit., p. 188: «As Memórias póstumas de Brás Cubas eram o rompimento tácito, mais completo e definitivo de Machado de Assis, com o Romantismo sob o qual nascera, crescera e se fizera escritor».

22 Tra i primi provvedimenti che contribuirono ad elevare lo status internazionale del Brasile vi è infatti il decreto di apertura dei porti alle nazioni amiche, emanato dal il 28 gennaio 1808 dal Principe reggente Dom João de Bragança. La misura era finalizzata a garantire la continuità del commercio (soprattutto con il Regno Unito), nonché delle relazioni diplomatiche e politiche. Sarà seguita da ulteriori provvedimenti come: la fondazione del Banco do Brasil (1808) e dell'Accademia Real Militar (1810), l'istituzione della stampa regia (1808), l'apertura di due scuole di Medicina e della Missão Artística Francesa (1816).

23 La città infatti continua ad essere la capitale dopo la dichiarazione di indipendenza, nel 1821, e per tutta la Prima Repubblica, che ha inizio nel 1889.

24 Cfr. CALMON, 2002: «A República fora feita no Rio de Janeiro: mas a sua energia civil, o alento económico, vieram de São Paulo. Joaquim Nabuco tratou-a de "republica militar cafezista...". Formara-se entretanto no 
sul outra mentalidade. Não era mais o liberalismo. Era o positivismo, a política "orgânica"”

25 I tratti impertinenti e superbi di cui fa ampio uso l'Autore sono stati giustificati da alcuni critici con l'intenzione di imitare, ridicolizzandolo, un modo di fare tipico della classe dominante dell'epoca: cfr. SCHWARZ, op. cit., p. 14.

26 La pesantezza della vita viene più volte contrapposta alla leggerezza e perfino alla "vitalità" del nuovo status post-mortem: "Uuro-lhes que essa orquestra da morte foi muito menos triste do que podia parecer. De certo ponto em diante chegou a ser deliciosa. A vida estrebuchava-me no peito, com uns ímpetos de vaga marinha, esvaía-se-me a consciência, eu descia à imobilidade física e moral, e o corpo fazia-se-me planta, e pedra e lodo, e coisa nenhuma» (Cap. I).

27 Su tutti cfr. SCHWARZ, op. cit., p. 23-24.

28 Il riferimento è al Capitolo CLX di Memorias Postumas de Bras Cubas, intitolato «Das negativas»: «[... Este último capítulo é todo de negativas. Não alcancei a celebridade do emplasto, não fui ministro, não fui califa, não conheci o casamento. Verdade é que, ao lado dessas faltas, coube-me a boa fortuna de não comprar o pão com o suor do meu rosto. Mais; não padeci a morte de Dona Plácida, nem a semi-demência do Quincas Borba. Somadas umas cousas e outras, qualquer pessoa imaginará que não houve míngua nem sobra, e, conseguintemente que saí quite com a vida. E imaginará mal; porque ao chegar a este outro lado do mistério, achei-me com um pequeno saldo, que é a derradeira negativa deste capítulo de negativas: -- Não tive filhos, não transmiti a nenhuma creatura o legado da nossa miséria».

29 MACHADO DE ASSIS, 2010, p. 14 e ss.

30 «Devo acrescentar que neste ponto manifesta-se às vezes uma opinião, que tenho por errônea: é a que só reconhece espírito nacional nas obras que tratam de assunto local, doutrina que, a ser exata, limitaria muito os cabedais da nossa literatura» Ivi, p. 26.

31 Di nuovo si ritorni alle osservazioni del saggio citato, Noticia da atual literatura brasileira. Instinto de nacionalidade: «Não se fazem aqui (falo sempre genericamente) livros de filosofia, de lingüística, de crítica histórica, de alta política, e outros assim, que em alheios países acham fácil acolhimento e boa extração; raras são aqui essas obras e escasso o mercado delas. $O$ romance podese dizer que domina quase exclusivamente. Não há nisto motivo de admiração nem de censura, tratando-se de um país que apenas entra na primeira mocidade, e esta ainda não nutrida de sólidos estudos».

32 LAJOLO, op. cit., p. 101.

33 Ibidem: «Criando suas personagens, Machado nada lhes perdoa: as mesquinharias pequenas e grandes; as indecisões, o oportunismo disfarçado; a falsa devoção; e a moral de fachada».

34 Poesia dal titolo «Ela», pubblicata il 12 gennaio 1855 nel periodico carioca Marmota Fluminense.

35 I liberti, inoltre, non erano equiparati alla popolazione libera. Fino al 1865 l'alforria, mediante il pagamento di denaro o liberamente concessa dal proprietario, poteva essere revocata dietro la semplice accusa di ingratitudine verso l'antico signore. Cfr. FAUSTO, 2012, p. 194.

36 Marisa LAJOLO, op. cit., p. 4.

37 La scelta di consegnare i figli allo Stato non spettava alla madre, bensì al «signore», ritenendosi ancora prevalente l'interesse economico del proprietario e la mancanza di autonomia privata della madre. A quest'ultimo spettava infatti un'indennità di 600 mila reais in caso di cessione del neonato allo Stato (art. 1, §1). Lo Stato avrebbe dovuto affidare i figli ad associazioni che si sarebbero dovute occupare della loro educazione, ma potevano anche esse usufruire dei loro «servizi gratuiti» fino all'età di 21 anni, oppure «affittare» tali servizi a terzi (art. 2, §1). La legge è consultabile nel sito del governo brasiliano: http://www.planalto.gov.br/ccivil_03/leis/LIM/LIM2040.htm

38 Convention between His Majesty and the Emperor of Brazil, for the Regulation and final Abolition of the African Slave Trade, firmata il 23 novembre 1826 a Rio de Janiero.

39 Si noti il passaggio di cui al paragrafo III nel quale il parlamento inglese denuncia esplicitamente l'ambiguità dei funzionari dell'Imperatore: «[...] it should not be lawful for the Subjects of the Emperor of Brazil to be concerned in the carrying on of the African Slave Trade under any Pretext or in any Manner whatever, and that the carrying on such Trade after that Period by any Person, Subject of His Imperial Majesty, should be deemed and treated as Piracy [...]»

40 Cfr. FAUSTO, op.cit., p. 167 e ss.

41 La Costituzione imperiale del 1824 introduceva, oltre ad una forma di parlamentarismo fortemente condizionata dal Poder Moderador della Corona, le garanzie tipiche della tradizione liberale. Nella formulazione di chiusura (l'art. 179) è contenuto un elenco dei principi e dei diritti fondamentali, tra i quali, oltre ai principi classici del liberalismo come quello di legalità, di irretroattività della legge 
penale e dell'indipendenza del potere giudiziario, è opportuno ricordare per la loro rilevanza: il divieto di discriminazione religiosa (V); il diritto dei detenuti ad un trattamento umano (XXI); il diritto di petizione e il ricorso al Potere Legislativo per le violazioni della Costituzione (XXX); la previsione dei soccorsi pubblici (XXXI); il diritto all'istruzione primaria gratuita a tutti i cittadini (XXXII).

42 Su questo bilancio della diffusione delle idee liberali oltreoceano, cfr. LOSURDO, 2005, p. 38 e ss.

43 LAJOLO, op. cit., p. 103.

44 Nell'incontro tra Brás Cubas e Quincas Borba il protagonista ascolta stupefatto le teorie del filosofo, lasciandosi coinvolgere dalla «chiarezza dell'esposizione» (Cap. CXVII). L'esattezza dell'Humanitismo viene dimostrata con una serie di deduzioni, a partire dalla divertente esaltazione delle virtù bellicose generate dall'invidia: «Nota que eu não faço do homem um simples veículo de Humanitas; não, ele é ao mesmo tempo veículo, cocheiro e passageiro; ele é o próprio Humanitas reduzido; daí a necessidade de adorar-se a si próprio. Queres uma prova da superioridade do meu sistema? Contempla a inveja. Não há moralista grego ou turco, cristão ou muçulmano, que não troveje contra o sentimento da inveja. O acordo é universal, desde os campos da Iduméia até o alto da Tijuca. Ora bem; abre mão dos velhos preconceitos, esquece as retóricas rafadas, e estuda a inveja, esse sentimento tão sutil e tão nobre. Sendo cada homem uma redução de Humanitas, é claro que nenhum homem é fundamentalmente oposto a outro homem, quaisquer que sejam as aparências contrárias. Assim, por exemplo, o algoz que executa o condenado pode excitar o vão clamor dos poetas; mas substancialmente é Humanitas que corrige em Humanitas uma infração da lei de Humanitas. O mesmo direi do indivíduo que estripa a outro; é uma manifestação da força de Humanitas. Nada obsta (e há exemplos) que ele seja igualmente estripado. Se entendeste bem, facilmente compreenderás que a inveja não é senão uma admiração que luta, e sendo a luta a grande função do gênero humano, todos os sentimentos belicosos são os mais adequados à sua felicidade. Daí vem que a inveja é uma virtude».

45 SCHWARZ, op. cit., p. 55 e ss.

46 MALAGUTI BATISTA, 2016, p. 299. Il principale riferimento dell'Autrice è alle teorie "igeniste", importate dalla tradizione del diritto penale portoghese ancora scarsamente secolarizzato, le quali trovarono continuità in Brasile anche dopo l'Indipendenza. Si faccia attenzione infatti ai seguenti passaggi: «É nesse cenário que, em Portugal, a religião se torna cada vez mais uma questão de Estado, associada às noções de ordem e segurança pública, ou seja, surgiam espaços de secularização sem ruptura com o poder clerical. [...] É esse positivismo que vai ocupar um espaço central na conjuntura da República brasileira. Nas nossas margens esse discurso médico impôs uma densa patologização dos africanos e dos afro-brasileiros bem como dos povos originários [... . O curioso é a recepção na periferia de uma cultura que nos deprecia e legitima nosso massacre. O dilema do Império Brasileiro, da Independência à República, era como conjugar liberalismo e escravidão e como dar conta das tentativas e desejos de protagonismo de um povo "inferior e degenerado", patológico ontologicamente» (p. 298-299).

47 L'ultima Costituzione brasiliana del 1988, con i suoi 250 articoli ed i 91 emendamenti che sono stati operati fino ad oggi, contiene una lunga e variegata serie di disposizioni in materia economica e sociale che hanno dato fondamento alla fortunata tesi della «Constituição dirigente» di CANOTILHO, 1982, p. 11.

48 Si fa riferimento alle mobilitazioni di piazza e alle divisioni interne alla magistratura e alla classe politica seguite all'apertura del processo di impeachment contro Dilma Rousseff e agli sviluppi dell'inchiesta "Lava-Jato». Quest'ultima, in particolare, ha portato alla luce un vasto schema di corruzione con riferimento gli appalti del colosso petrolifero a partecipazione pubblica (Petrobras), nella quale sono risultati coinvolti gran parte dei partiti politici brasiliani. Tra le opposte ricostruzioni delle vicende che hanno scandito l'inchiesta giudiziaria si veda la riflessione di COUTINHO i AMATUZZI, 2016. 


\title{
POWER AND POLITICS IN THE CONSTITUTIONAL EXPERIENCE OF POST- COLONIAL BRAZIL. A REFLECTION FROM A TITLE BY MACHADO DE ASSIS
}

\begin{abstract}
The complexity of relations of power can be discerned in many of Machado de Assis' works, through writings that portray the society of Rio de Janeiro in a moment when the Brazilian State was still taking steps towards a permanent institutional arrangement. In this context, the position of Machado de Assis is twice as significant for a European scholar: on the one hand, it is testimony of the tensions that developed regarding the process of dissemination of the political and philosophical theories emanating from the Old Continent; on another hand, it identifies the Brazilian reality as a product of the contradiction between the archaic and the modern, between the old canons and the progress driven by individual ambitions.
\end{abstract}

Keywords: Law and literature. Post-Colonial studies. Academic dialogue Brazil-Italy. Circulation and reception of western legal models.

Submetido: 7 out. 2016

Aprovado: 20 out. 2016 